\title{
Histopathological growth distribution of ductal carcinoma in situ: tumor size is not "one size fits all"
}

\author{
Thomas J. O’Keefe ${ }^{1 \wedge}$, Olivier Harismendy ${ }^{2}$, Anne M. Wallace ${ }^{1}$ \\ ${ }^{1}$ Division of Breast Surgery and the Comprehensive Breast Health Center, University of California San Diego, La Jolla, CA, USA; ${ }^{2}$ Moores Cancer \\ Center and Division of Biomedical Informatics, Department of Medicine, University of California San Diego, La Jolla, CA, USA \\ Contributions: (I) Conception and design: TJ O'Keefe, AM Wallace; (II) Administrative support: TJ O'Keefe; (III) Provision of study materials \\ or patients: TJ O'Keefe, AM Wallace; (IV) Collection and assembly of data: TJ O'Keefe; (V) Data analysis and interpretation: TJ O'Keefe; (VI) \\ Manuscript writing: All authors; (VII) Final approval of manuscript: All authors. \\ Correspondence to: Thomas J. O’Keefe. Mailbox 0819, 3855 Health Sciences Dr, La Jolla, CA 92037, USA. Email: tokeefe@health.ucsd.edu.
}

\begin{abstract}
Background Ductal carcinoma in situ (DCIS) is a group of preinvasive breast neoplasms. Studies have shown excellent survival among patients with lumpectomy-amenable disease. Patients requiring mastectomy have been less well characterized. We aim to characterize this cohort and identify whether growth distribution pattern is associated with sentinel lymph node involvement at time of surgery or subsequent development of metastatic disease.

Methods: Patients were identified using local cancer registry data and were chart reviewed using electronic medical records. Growth pattern was classified as unifocal, multifocal, or diffuse. Chi-squared, Analysis of Variance (ANOVA), and Kaplan-Meier analyses were performed.

Results: Two hundred and twenty-six patients were identified with median age at diagnosis 49 and follow up 7.1 years. 42 had unifocal, 51 had multifocal and 20 had diffuse lesions. 3/20 patients with diffuse type lesions developed subsequent distant metastatic disease, while none of the patients with unifocal or multifocal lesions did. 1/20 patients with diffuse and 2/51 with multifocal disease had sentinel lymph node involvement (SLNI) at surgery. Tumor extent was not associated with sentinel lymph node involvement or distant metastatic disease $(\mathrm{P}=0.2$, Kaplan-Meier analysis) but growth pattern was $(\mathrm{P}=0.01)$. It was also associated on Kaplan-Meier with development of distant metastatic disease alone $(\mathrm{P}=0.01)$.

Conclusions: Patients with diffuse growth pattern DCIS were more likely to have SLNI or development of distant metastatic disease. Our findings suggest that patients with diffuse type lesions are at greater risk of metastatic disease and therefore breast cancer death from DCIS. Optimal therapy for these patients will need further elucidation.
\end{abstract}

Keywords: Ductal carcinoma in situ (DCIS); breast cancer metastasis; mortality; outcomes

Submitted Aug 30, 2021. Accepted for publication Jan 07, 2022.

doi: $10.21037 /$ gs-21-599

View this article at: https://dx.doi.org/10.21037/gs-21-599

\section{Introduction}

Ductal carcinoma in situ (DCIS) is a commonly diagnosed neoplastic breast process that encompasses a heterogeneous group of lesions which vary with respect to histopathologic features and outcomes (1). Mastectomy represented the initial standard of care for DCIS because of its potential to progress to metastatic disease $(2,3)$, but after randomized trials for invasive breast cancer demonstrated that mastectomy did not result in a difference in outcomes relative to lumpectomy with adjuvant radiation therapy, also known as breast conservation therapy, lumpectomy was adopted for the treatment of DCIS $(2,3)$ since it

\footnotetext{
^ ORCID: 0000-0003-1439-8569.
} 
was postulated to represent less aggressive disease (4). Importantly, the subsequent DCIS trials investigating the use of adjuvant radiation and endocrine therapy were performed on patients with lumpectomy-amenable disease, not disease requiring mastectomy, despite mastectomies representing between $27-56 \%$ of the surgeries received by DCIS patients (5-11).

Due to the previously mentioned low breast cancer mortality rates observed among patients with lumpectomyamenable DCIS, treatment and research currently emphasize the prevention of recurrence. Despite this, interest within the research community in the ability of what appears at the microscopic level to be in situ disease to subsequently metastasize to distant sites is based on indirect evidence from multiple sources. The clinical and biological evidence supporting this phenomenon has been summarized by Narod et al. (12).

DCIS size is a known independent predictor of residual disease and is utilized in prognostic tools such as the Van Nuys Prognostic Index $(13,14)$. Multifocality, when multiple foci of disease arise within the same quadrant of the breast, has been linked with poorer prognosis among patients with DCIS relative to patients with unifocal disease, when there is a single contiguous focus of DCIS $(15,16)$. Swedish pathologist Tibor Tot identified a third spatial configuration of DCIS which he called diffuse, referring to tumors spread out over a large area with no distinct tumor edge, referring to histopathologic findings rather than clinical or imaging findings (17). He later demonstrated that this diffuse growth distribution as well as multifocal disease were associated with breast cancer mortality relative to unifocal lesions in an analysis including mostly mixed invasive and in situ but some pure in situ lesions (18). The American Joint Committee on Cancer guidelines advises utilizing the largest contiguous focus of disease for tumor sizing, so these lesions are expected to be coded in cancer registries by the size of their largest contiguous focus (19). However for patients with diffuse and multifocal DCIS, it is unclear if this represents the most useful measure of size for the purposes of risk stratification. Diffuse DCIS in particular, which is known to have poor outcomes for invasive and mixed invasive-in situ cancers, may have a small "largest contiguous" focus of disease, despite having a large span of disease throughout the breast. If this were the case then analyses performed on DCIS using cancer registry data may lead to misleading conclusions about the impact of DCIS size.

We sought to characterize patients undergoing mastectomy for DCIS at our institution using both our local cancer registry data and chart review in this cohort study. We also aimed to investigate the effects of largest contiguous focus of DCIS as well as growth distribution of DCIS on sentinel lymph node involvement or the development of subsequent metastatic disease (SLNI/ $\mathrm{DMD}$ ) in patients diagnosed at our institution with pure DCIS who underwent mastectomy for their disease.

We present the following article in accordance with the STROBE reporting checklist (available at https:// gs.amegroups.com/article/view/10.21037/gs-21-599/rc).

\section{Methods}

A list of in situ and invasive breast cancer lesions diagnosed at UCSD from January 1, 2000 to March 21, 2019 was obtained from the UCSD cancer registry. There were 7,755 breast cancer events among 7,290 patients (Figure 1). Registry data was used to only include patients classified as female with first cancer of breast origin, DCIS with or without microinvasive disease, who underwent mastectomy $(\mathrm{N}=259)$. On chart review, 10 additional patients were excluded because they had non-microinvasive invasive disease, 21 were excluded because their pathology reports from the mastectomy were missing, and 2 were excluded because they had a past history of invasive breast cancer that had not been documented in the cancer registry.

The remaining 226 patients were reviewed for date of mastectomy, largest contiguous focus of disease, overall disease extent, growth distribution, presence or absence of microinvasive disease, grade of disease, estrogen receptor (ER), progesterone receptor (PR) and HER2 statuses, surgical margins after mastectomy, whether the patients underwent unilateral or bilateral mastectomy, lymph node evaluation and number of lymph nodes involved and uninvolved with disease, whether endocrine therapy (ET) was administered, whether RT was administered, whether the patient developed metastatic breast cancer, and whether the patient died of breast cancer.

The growth pattern of the lesions were classified into the following groups: unifocal, multifocal, and diffuse. Pathology reports were the primary means of identification of the type of growth pattern. In cases in which the pathology report stated that the lesion was unifocal, it was classified as such. In cases in which the lesion had descriptive characteristics of numerous, scattered, or a multitude of small or microscopic foci, with or without an associated larger focal lesion or lesions, the lesion was classified as diffuse. In cases in which the previously 


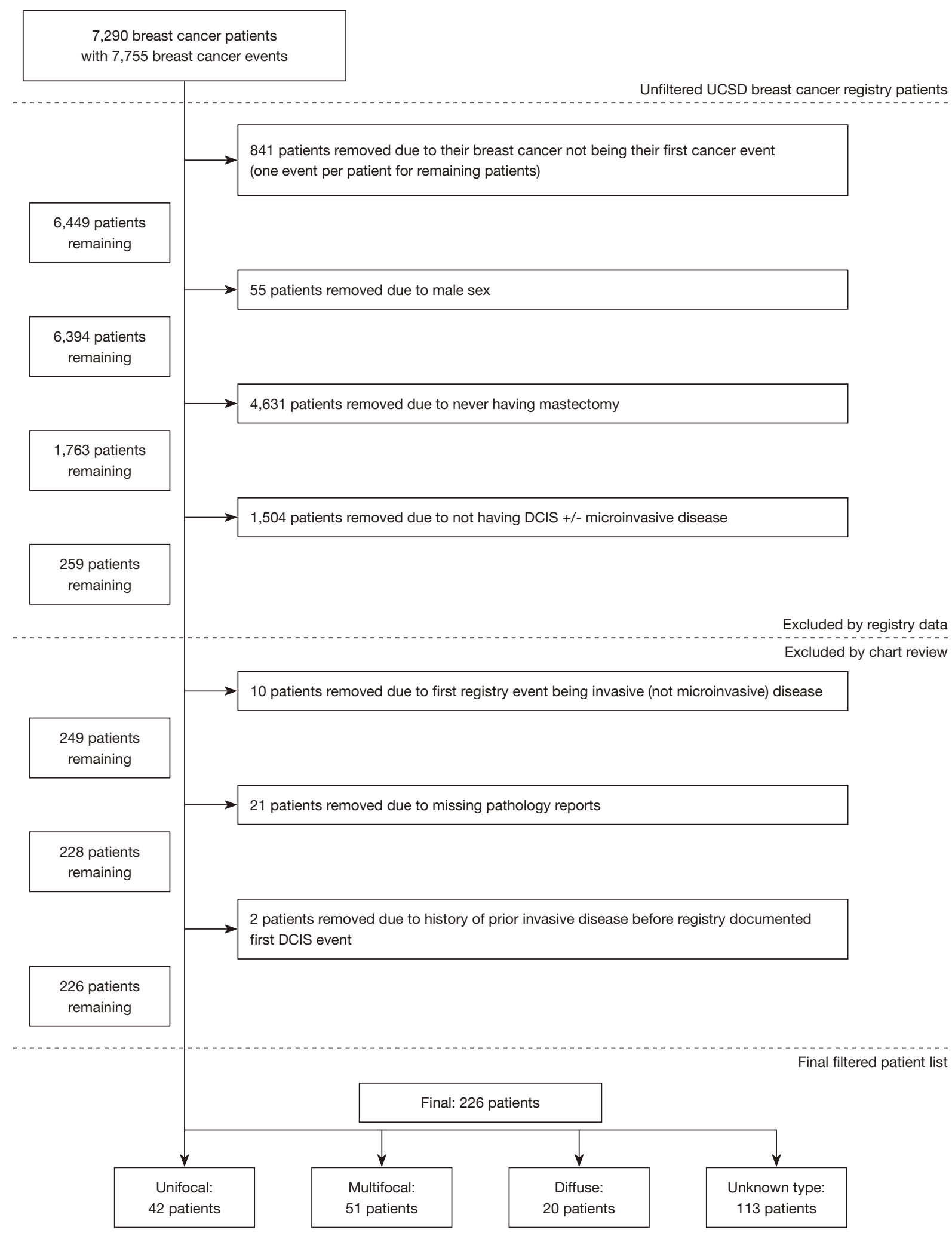

Figure 1 Application of exclusion criteria to initial UCSD cancer registry data. UCSD, University of California San Diego; DCIS, ductal carcinoma in situ. 
mentioned descriptive terms were not applied and the lesion was classified on the pathology report as multifocal or multicentric (multiple foci present in different quadrants of the breast) the lesion was classified as multifocal.

Size was classified by both largest contiguous size and overall extent of disease (EOD) by pathology reports. For unifocal lesions, the documented size in the report was used for both the largest contiguous size and the EOD. For patients with multifocal or diffuse disease, if there was mention of a measurement regarding the largest contiguous focus of disease, this was used as such, and if there was mention of a measurement of the total spread or extent over which the foci were dispersed, this measurement was utilized for the EOD. In patients for whom there was no identifiable classifier of growth pattern, any size described in the report was documented separately from the previously mentioned size and EOD.

\section{Statistical analysis}

Growth pattern groups were compared using complete case analysis with Fisher's exact test, Chi-squared test, and ANOVA, with global differences in ANOVA being examined by Tukey's range test. Kaplan-Meier estimator was used to generate curves for SLNI/DMD among groups and log-rank test was used to compare groups. $\mathrm{P}$ values were calculated as two-sided and statistical significance was declared for $\mathrm{P}$ less than 0.05 . Loss to follow up was dictated by the data available in the cancer registry and electronic medical records and was not accounted for. All statistical analyses was performed in $\mathrm{R}$ (version 3.5.1, R Foundation for Statistical Computing, Vienna, Austria) using RStudio (Version 1.1.463) and packages "tidyverse" (Version 1.3.0), "survival" (Version 3.1-7) and "survminer" (Version 0.4.6).

\section{Ethical statement}

The study was conducted in accordance with the Declaration of Helsinki (as revised in 2013). The study was approved by the UCSD Human Research Protections Program (IRB \#181461). Informed consent was not obtained due to the large number of patients involved, which, coupled with the fact that contact information was likely unavailable or outdated for many of the patients given the prolonged period over which the study was conducted, made obtaining consent for all patients impractical.

\section{Results}

\section{Overall population characterization}

A total of 226 patients met the inclusion criteria, but only 113 among them could be classified in terms of growth distribution pattern after review of pathology reports. 42 (37.2\%) had unifocal, 51 (45.1\%) had multifocal, and 20 (17.7\%) had diffuse growth distribution lesions (Table 1). Median follow up time was 7.1 years (interquartile range 4.1-11.7 years). Median age at diagnosis was 49 years (4357 years).

\section{Differences among growth distribution cohorts}

When grouped by growth distribution, several differences in covariates were noted. On Chi-squared analysis, there was a significant difference in the reason for mastectomy $(\mathrm{P}<0.0001)$ with patients having unifocal lesions being more likely to have a mastectomy related to prophylaxis and less likely to undergo mastectomy for an inability to achieve negative margins. There was a significant difference among groups on ANOVA $(\mathrm{P}=0.02)$ with respect to the largest contiguous focus of disease, with patients having unifocal disease having larger contiguous foci of disease than patients with multifocal disease $(\mathrm{P}=0.03)$. Similarly, there was a significant difference among groups with respect to EOD $(\mathrm{P}<0.0001)$, with diffuse growth distribution lesions having greater EOD than both multifocal $(\mathrm{P}=0.0005)$ and unifocal $(\mathrm{P}=0.0001)$ type lesions.

\section{Differences in outcome by largest contiguous focus of disease, extent of disease, and growth distribution}

Either sentinel lymph node involvement or presence of distant metastatic disease (SLNI/DMD) were identified in $9(4.0 \%)$ of the 226 patients (Table 2), including 5 (2.2\%) patients who developed distant metastatic disease and 4 $(1.8 \%)$ who were found to have isolated tumor cells or micrometastases on sentinel lymph node biopsy during one or more of their DCIS procedures. Median age at diagnosis for the SLNI/DMD cases was lower than the overall group at 42 years. Among the 5 patients who developed distant metastatic disease, all 3 of the patients with lesions that could be classified were diffuse growth distribution. Among the patients who developed distant metastases, none of the patients had an intervening in breast invasive or in situ 
Table 1 Characterization of the patients in the cohort of patients undergoing mastectomy for DCIS grouped by their pattern of growth pattern

\begin{tabular}{|c|c|c|c|c|}
\hline & Unifocal & Multifocal & Diffuse & Unclassifiable \\
\hline Total & $42(100.0 \%)$ & $51(100.0 \%)$ & $20(100.0 \%)$ & $113(100.0 \%)$ \\
\hline \multicolumn{5}{|l|}{ Age, years } \\
\hline$\leq 45$ & $14(33.3 \%)$ & $22(43.1 \%)$ & $8(40.0 \%)$ & $40(35.4 \%)$ \\
\hline $45-60$ & $14(33.3 \%)$ & $17(33.3 \%)$ & $9(45.0 \%)$ & $40(35.4 \%)$ \\
\hline Bilateral disease & $1(2.4 \%)$ & $3(5.9 \%)$ & $2(10.0 \%)$ & $0(0.0 \%)$ \\
\hline Lumpectomy first & $6(14.3 \%)$ & $17(33.3 \%)$ & $7(35.0 \%)$ & $35(31.0 \%)$ \\
\hline Median time lumpectomy to mastectomy (days) & 97.5 & 70 & 84 & 71.5 \\
\hline \multicolumn{5}{|l|}{ Largest contiguous focus, $\mathrm{cm}$} \\
\hline Missing & $3(7.1 \%)$ & $24(4.7 \%)$ & $16(80.0 \%)$ & $\mathrm{N} / \mathrm{A}^{\dagger}$ \\
\hline \multicolumn{5}{|l|}{ Extent, cm } \\
\hline$\leq 4$ & $26(6.2 \%)$ & $3(5.9 \%)$ & $1(5.0 \%)$ & $\mathrm{N} / \mathrm{A}^{\dagger}$ \\
\hline $4-7$ & $9(21.4 \%)$ & 7 (13.7\%) & 4 (20.0\%) & $\mathrm{N} / \mathrm{A}^{\dagger}$ \\
\hline$>7$ & $4(9.5 \%)$ & $6(11.8 \%)$ & 10 (50.0\%) & $\mathrm{N} / \mathrm{A}^{\dagger}$ \\
\hline Missing & $3(7.1 \%)$ & $35(68.6 \%)$ & $5(25.0 \%)$ & $\mathrm{N} / \mathrm{A}^{\dagger}$ \\
\hline \multicolumn{5}{|l|}{ Estrogen receptor } \\
\hline \multicolumn{5}{|l|}{ Progesterone receptor } \\
\hline Negative & $8(19.0 \%)$ & $12(23.5 \%)$ & $6(30.0 \%)$ & $39(34.5 \%)$ \\
\hline Borderline & $0(0.0 \%)$ & $0(0.0 \%)$ & $0(0.0 \%)$ & $1(0.9 \%)$ \\
\hline Missing & $2(4.8 \%)$ & 8 (15.7\%) & $1(5.0 \%)$ & 22 (19.5\%) \\
\hline \multicolumn{5}{|l|}{ HER2 receptor } \\
\hline Positive & $12(28.6 \%)$ & $12(23.5 \%)$ & $5(25.0 \%)$ & $36(31.9 \%)$ \\
\hline Negative & $16(38.1 \%)$ & $17(33.3 \%)$ & $9(45.0 \%)$ & $34(30.1 \%)$ \\
\hline Equivocal & 7 (16.7\%) & 5 (9.8\%) & $0(0.0 \%)$ & $2(1.8 \%)$ \\
\hline Missing & $7(16.7 \%)$ & $17(33.3 \%)$ & $6(30.0 \%)$ & $51(45.1 \%)$ \\
\hline Bilateral mastectomy & $14(33.3 \%)$ & $15(29.4 \%)$ & $4(20 \%)$ & $36(31.9 \%)$ \\
\hline
\end{tabular}

Table 1 (continued) 
Table 1 (continued)

\begin{tabular}{|c|c|c|c|c|}
\hline & Unifocal & Multifocal & Diffuse & Unclassifiable \\
\hline \multicolumn{5}{|l|}{ Reason for mastectomy } \\
\hline Extent of disease & $27(64.3 \%)$ & $26(51.0 \%)$ & $13(65.0 \%)$ & $48(42.5 \%)$ \\
\hline Positive margins & $4(9.5 \%)$ & $17(33.3 \%)$ & $7(35.0 \%)$ & $25(22.1 \%)$ \\
\hline Prophylaxis & $8(19.0 \%)$ & $5(9.8 \%)$ & $0(0.0 \%)$ & $12(10.6 \%)$ \\
\hline Missing & $1(2.4 \%)$ & $3(5.9 \%)$ & $0(0.0 \%)$ & $14(12.4 \%)$ \\
\hline \multicolumn{5}{|l|}{ Grade } \\
\hline Low & $6(14.3 \%)$ & $6(11.8 \%)$ & $0(0.0 \%)$ & $11(9.7 \%)$ \\
\hline Intermediate (or low-intermediate) & $15(35.7 \%)$ & $13(25.5 \%)$ & $8(40.0 \%)$ & $31(27.4 \%)$ \\
\hline Sentinel node biopsy & $39(92.9 \%)$ & $50(98.0 \%)$ & $19(95.0 \%)$ & $106(93.8 \%$ \\
\hline Lymph node metastasis & $0(0.0 \%)$ & $2(3.9 \%)$ & $1(5.0 \%)$ & $1(0.9 \%)$ \\
\hline Microinvasion & $5(11.9 \%)$ & $7(13.7 \%)$ & $2(10.0 \%)$ & $20(17.7 \%)$ \\
\hline Endocrine therapy & $6(14.3 \%)$ & $13(25.5 \%)$ & $1(5.0 \%)$ & $20(17.7 \%)$ \\
\hline Radiation therapy & $0(0.0 \%)$ & $0(0.0 \%)$ & $0(0.0 \%)$ & $0(0.0 \%)$ \\
\hline Distant metastasis & $0(0.0 \%)$ & $0(0.0 \%)$ & $3(15.0 \%)$ & $2(1.8 \%)$ \\
\hline
\end{tabular}

${ }^{\dagger}$, listed sizes could not be classified as size or extent for patients of unknown growth pattern; among patients with a documented "size", the median "size" was $2.2 \mathrm{~cm}$ and the interquartile range was $1.0-4.8 \mathrm{~cm}$. DCIS, ductal carcinoma in situ.

recurrence. None of the patients who developed metastatic disease had involvement of their sentinel lymph node biopsy at the time of surgery, and none of the patients who had SLNI at the time of surgery went on to develop metastatic disease. None of the patients who developed distant metastatic disease had positive margins; 1 patient who had SLNI had positive margins.

Kaplan Meier estimators were calculated with SLNI/ $\mathrm{DMD}$ as the event of interest. When grouped by largest contiguous size into $\leq 1.5 \mathrm{~cm}, 1.5-4 \mathrm{~cm}$, or $>4 \mathrm{~cm}$, there was no significant difference among the groups $(\mathrm{P}=0.48)$. When grouped by the EOD using largest focus of disease for unifocal lesions into $\leq 4 \mathrm{~cm}, 4-7 \mathrm{~cm}$, and $>7 \mathrm{~cm}$, there was again no significant difference among groups $(\mathrm{P}=0.2)$. When grouped by the growth pattern, there were differences among the groups $(\mathrm{P}=0.01)$ with patients with diffuse growth distribution being more likely to have SLNI/DMD (Figure 2). Kaplan-Meier analysis applied exclusively to the outcome of development of distant metastatic disease did not achieve significance with respect to largest contiguous focus of disease $(\mathrm{P}=0.59)$, was borderline significant for EOD $(\mathrm{P}=0.06)$, and was significant for growth pattern $(\mathrm{P}=0.01)$.

\section{Discussion}

Here we report an association between diffuse growth distribution type DCIS lesions with SLNI/DMD and development of distant metastatic disease. While our results should be interpreted with caution given the small cohort size, this finding is novel and has important clinical implications. Presently, standard of care for DCIS involves excision of the lesion with negative margins, with adjuvant RT and ET considered to reduce recurrences and progression to invasive disease. Our results suggest that patients with diffuse growth distribution DCIS are at higher risk of systemic disease and have worse prognoses. The optimal management of these patients may therefore differ from that for the more common unifocal and multifocal DCIS. 


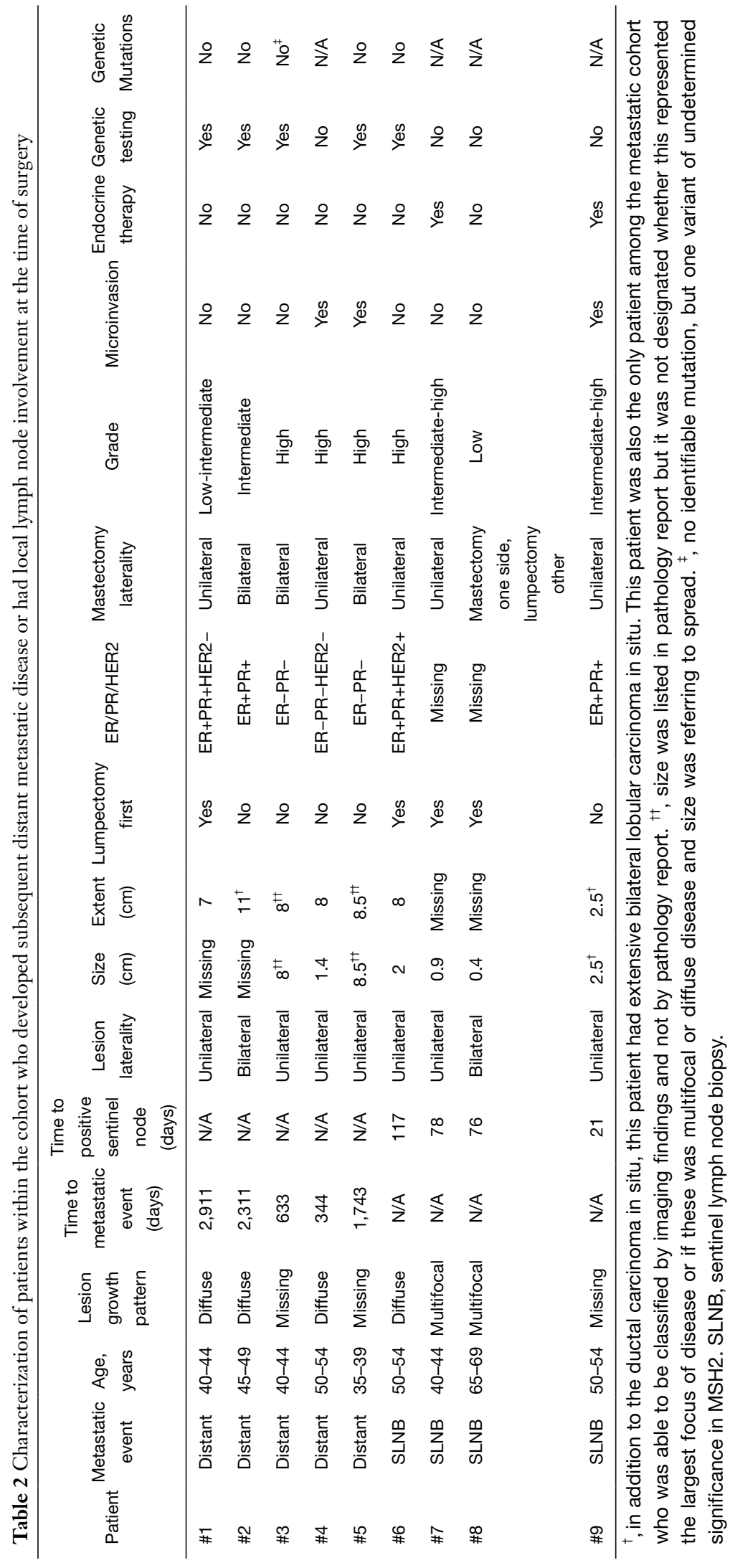




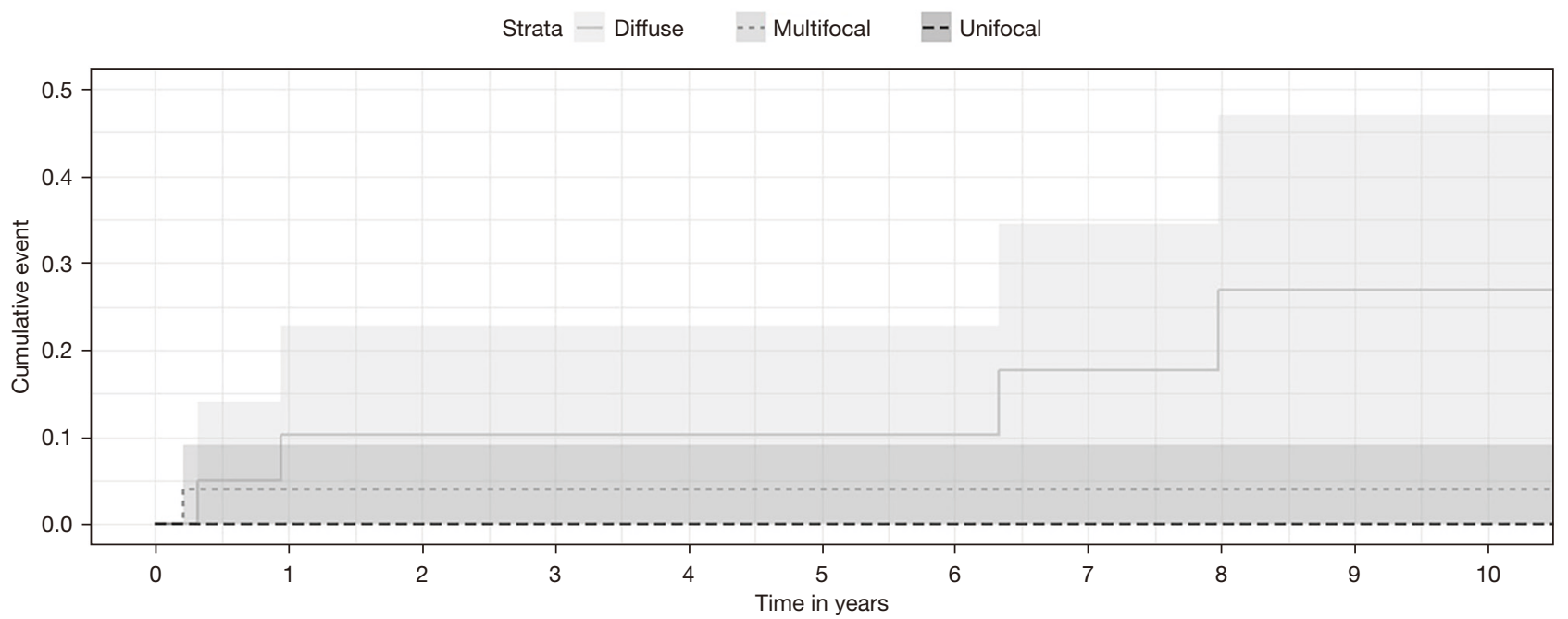

\begin{tabular}{|c|c|c|c|c|c|c|c|c|c|c|c|}
\hline \multirow{5}{*}{$\begin{array}{l}\frac{\pi}{\pi}= \\
\frac{\pi}{4}= \\
\text { 心 }=\end{array}$} & \multicolumn{11}{|c|}{ Number at risk: $\mathrm{n}(\%)$} \\
\hline & $20(100)$ & $17(85)$ & $16(80)$ & $16(80)$ & $14(70)$ & $13(65)$ & $12(60)$ & $11(55)$ & $8(40)$ & $7(35)$ & 7 (35) \\
\hline & $51(100)$ & 47 (92) & $42(82)$ & $37(73)$ & $31(61)$ & $27(53)$ & $23(45)$ & $19(37)$ & $19(37)$ & $16(31)$ & $14(27)$ \\
\hline & $41(100)$ & $40(98)$ & $35(85)$ & $31(76)$ & $22(54)$ & $11(27)$ & $6(15)$ & $5(12)$ & $3(7)$ & $2(5)$ & $1(2)$ \\
\hline & 0 & 1 & 2 & 3 & 4 & $\begin{array}{c}5 \\
\text { rime in yea }\end{array}$ & 6 & 7 & 8 & 9 & 10 \\
\hline
\end{tabular}

Figure 2 Kaplan Meier estimator for development of SLNI/DMD by growth distribution. SLNI/DMD, sentinel lymph node involvement or distant metastatic disease.

Breast cancer mortality, which is most commonly mediated by metastatic disease and its complications (20), has not been a focus of DCIS research because treatment has not been shown to affect this outcome $(2,21)$. We believe that this is at least partly related to the issue of treating DCIS, which represents a heterogeneous group of diseases, as a single entity. Our results suggest that diffuse growth distribution DCIS represents a subgroup of DCIS with a worse prognosis, not just for progression to invasion but more importantly for progression to metastatic disease. Tibor Tot, a Swedish pathologist, characterized DCIS lesions into unifocal, multifocal and diffuse type lesions in 2005 and showed higher recurrence rates among patients with more extensive disease (22). He subsequently showed in 2007 and 2010 that diffuse growth distribution of invasive components of breast cancer lesions were associated with higher rates of lymph node metastasis, and in 2011 that combined extent of invasive and in situ components, as well as presence of multifocal or diffuse growth distribution of breast neoplasms was associated with lower overall survival and breast cancer specific survival relative to smaller extent and unifocal type lesions, respectively, in a cohort that included both invasive, in situ, and mixed lesions $(17,18,23)$. While Tot's findings are of great interest from a prognostication standpoint for invasive lesions, the treatment implications are less clear as invasive disease is presently treated with systemic therapy as part of standard of care. For DCIS, however, the only approved systemic therapy is ET for hormone receptor positive disease.

While we designed our study to group SLNI at time of surgery and distant metastatic disease together as the combined outcome SNLI/DMD, our findings suggest that the process by which these two events are mediated are different. There was no overlap between the two groups with none of the patients with positive sentinel nodes developing subsequent distant metastatic disease and none of the patients who developed distant metastatic disease having had positive sentinel lymph nodes, though one patient (Patient \#1 in Table 2) had a small $0.2 \mathrm{~mm}$ focus of keratin positive staining identified in one of her six sentinel lymph nodes, though the report reflected that this was not felt to be isolated tumor cells or micrometastatic disease. SLNI may be mediated by iatrogenic release of DCIS cells secondary to an invasive procedure such as needle biopsy or lumpectomy, with some evidence that the likelihood of lymph node involvement by isolated tumor cells or micrometastatic disease in DCIS is proportional to the invasiveness of the procedure preceding the $\operatorname{SLNB}(24,25)$. 


\begin{tabular}{|c|c|c|c|}
\hline & Unifocal & Multifocal & Diffuse \\
\hline \multicolumn{4}{|l|}{ Early } \\
\hline \multicolumn{4}{|l|}{ Late } \\
\hline Number of cells & $+/++/+++$ & $+/++/+++$ & +++ \\
\hline $\begin{array}{l}\text { Cellular } \\
\text { heterogeneity }\end{array}$ & + & ++ & ++ \\
\hline Invasive potential & + & ++ & +++ \\
\hline $\begin{array}{l}\text { Distant metastatic } \\
\text { potential }\end{array}$ & + & ++ & +++ \\
\hline
\end{tabular}

Figure 3 Models of DCIS growth distribution patterns and associations with metastatic potential. For each growth pattern a schematic breast ductal tree is displayed at different time of DCIS progression (early and late). Independent lesions of different sizes are indicated by circle of varying diameters. Shades of grey illustrate phenotypic heterogeneity of the lesion (circles) or along the tree (diffuse disease). Dashed circles and budding branches illustrate potential for invasion. The relative properties of each growth pattern are summarized below. DCIS, ductal carcinoma in situ.

Supporting this explanation is the fact that three of the four patients who had positive lymph node involvement in our study had lumpectomy prior to their mastectomy. It is therefore important to note that in Kaplan-Meier analysis diffuse growth distribution remained significant even when the outcome was specifically development of distant metastatic disease, without categorizing it together with sentinel lymph node involvement. While none of the patients with lymph node metastases in our study were treated with adjuvant chemotherapy, it is worth noting that such clinical scenarios can be categorized as Stage IIA lesions. Such patients could then potentially be considered for adjuvant chemotherapy depending on the subtype, though given the non-overlap in our cohort of development of subsequent metastatic disease with identification of sentinel lymph node metastasis, our results do not provide evidence to support this. Another limitation is that due to the small sample size and small number of events of interest, we were unable to construct a proportional hazards model for development of subsequent metastatic disease as such a model did not converge.

A fascinating question arising from this study is what the mechanism of metastatic spread among these patients with diffuse growth distribution DCIS is. While our outcomesbased results do not provide evidence for any particular mechanism, we propose a model by which this phenomenon occurs. If growth distribution were visualized as a spectrum, with one end of the spectrum representing purely unifocal, contiguous lesions, and the opposite end of the spectrum representing purely diffuse growth distribution DCIS lesions with scattered small foci that do not form a contiguous mass (Figure 3), then multifocal disease would fall between these distributions on the spectrum type. We hypothesize that any two DCIS cells at a given distance within a unifocal lesion are the result of the proliferation of a common ancestor clone, in contrast to two equivalently spaced DCIS cells from a diffuse growth distribution lesion which more likely have originated from independent transformations. In turn, cells in diffuse growth distribution lesions may have a greater degree of phenotypic heterogeneity owing to the differences in origin. As a result a greater proportion of cells in diffuse growth distribution lesions would be expected to have the ability to adapt to a new tumor microenvironmental niche after metastasizing. It is also possible that this phenotypic heterogeneity may result in increased invasive potential, as small foci scattered throughout the lesion may develop the ability to invade rather than a single proliferating cell line producing an invasive focus. Tot demonstrated that among patients with neogenetic diffuse DCIS there is a higher local recurrence rate $(27 \%)$ than among diffuse, non- 
neogenetic (14\%), multifocal (14\%) and unifocal (2\%), where neogenetic refers to evidence of abnormalities in arborization of the lobe (22).

Of note, while we believe that the association of diffuse growth distribution lesions with metastatic disease is mediated by the above proposed mechanism, our findings regarding increased risk of metastatic disease among patients with diffuse growth distribution DCIS are not dependent on this model. An alternative explanation for these findings would be that in patients with diffuse growth distribution lesions, there are more likely to be missed foci of microinvasive disease. Narod and Giannakeas have previously demonstrated using SEER data that patients undergoing unilateral mastectomy did not have a survival benefit over patients undergoing lumpectomy, whereas patients undergoing lumpectomy with adjuvant radiation therapy did $(21,26)$. Problematically, since SEER registrars are instructed to code largest contiguous focus of disease rather than overall extent of disease, this would lead to patients with diffuse DCIS to being matched on the basis of their largest focus of disease, which could be quite small, and would therefore likely mismatch these patients. Furthermore, SEER registrars are instructed to recode patients who suffer breast cancer mortality after DCIS without an intervening invasive lesion as having initially had an invasive lesion, which is inherently problematic in terms of using SEER to estimate breast cancer mortality after a diagnosis of pure DCIS (27). Furthermore, we showed in a recent analysis including patients undergoing both unilateral and bilateral mastectomy that while in a univariate model mastectomy did not reduce breast cancer mortality, in the multivariate model there was a significant reduction, though this conclusion is still limited by the previously mentioned shortcomings of SEER data (28).

The greatest strength of our study is the quality of the data. All covariate data was obtained from chart review by the same individual. The greatest limitation is the small sample size combined with the rarity of the outcome of interest. Another significant limitation was the lack of centralized pathology review, which was due to the large number of missing primary specimens from the cohort, including those of all of the patients who developed distant metastatic disease. An additional limitation is the generalizability of the study, as all of our data were obtained from a single, local cancer database leading to a small sample size. Another limitation is the large number of missing largest contiguous size data for the diffuse type group. We were limited to what was available in the pathology reports since only representative specimens from the original surgical specimen are stored long term, preventing us from estimating both largest contiguous size and extent of disease.

While our results should be interpreted with caution, one clinical implication that might be drawn from this study is that consideration for the consistent documentation of not only the largest contiguous focus of disease, as is currently done by cancer registries as guided by SEER, but also of the growth distribution pattern and associated EOD. This would allow for large database study of the implications of these factors in longitudinal follow up of patients treated for DCIS.

Prospective, ideally randomized studies would be needed to confirm that the diffuse growth distribution cohort would benefit from adjuvant ET, but such a study would require a large multi-institutional effort due to the rarity of both large diffuse growth distribution DCIS lesions and breast cancer mortality after a pure DCIS diagnosis. Since HER2 receptor monoclonal antibodies are not presently considered part of standard of care options for DCIS, trastuzumab cannot be considered for HER2 positive diffuse growth distribution lesions from this study alone, though our results suggest this may confer a breast cancer mortality benefit and that, as with ET, further investigation is warranted. Strong prospective data would be necessary to suggest systemic chemotherapy for large diffuse growth distribution DCIS of triple negative molecular phenotype due to the more significant risks associated with chemotherapy regimens. However given the high rate of development of distant metastatic disease in our cohort, it is reasonable to hypothesize that this subgroup may benefit from such an escalation of therapy. In conclusion, the clinical implications of the influence of diffuse growth distribution on pure DCIS lesions in terms of development of SLNI/DMD and in particular development of distant metastatic disease is both novel and important given the significant risk posed to patients in spite of the infrequency of these events. We believe that subsets of DCIS patients, such as those with diffuse growth distribution lesions, represent cohorts for whom the focus on outcomes should not be on recurrence but rather metastatic disease and subsequent breast cancer mortality.

\section{Acknowledgments}

This work was supported by the UCSD Cancer Registry. And in particular, Marcy Anderson, whom we acknowledge for procuring the initial data set used for selection of 
patients involved in the study.

Funding: None.

\section{Footnote}

Reporting Checklist: The authors have completed the STROBE reporting checklist. Available at https:// gs.amegroups.com/article/view/10.21037/gs-21-599/rc

Data Sharing Statement: Available at https://gs.amegroups. com/article/view/10.21037/gs-21-599/dss

Peer Review File: Available at https://gs.amegroups.com/ article/view/10.21037/gs-21-599/prf

Conflicts of Interest: All authors have completed the ICMJE uniform disclosure form (available at https://gs.amegroups. com/article/view/10.21037/gs-21-599/coif). OH reports that during the research presented, he was paid consulting fees for expert witness service. He received dividends from Novartis and Sanofi, and funding from the National Cancer Institute, Padres Pedal the Cause and the UC Office of the President. After the completion of the research, he became an employee and received stocks options from Zentalis Pharmaceuticals. The other authors have no conflicts of interest to declare.

Ethical Statement: The authors are accountable for all aspects of the work in ensuring that questions related to the accuracy or integrity of any part of the work are appropriately investigated and resolved. The study was conducted in accordance with the Declaration of Helsinki (as revised in 2013). The study was approved by the UCSD Human Research Protections Program (IRB \#181461). Informed consent was not obtained due to the large number of patients involved, which, coupled with the fact that contact information was likely unavailable or outdated for many of the patients given the prolonged period over which the study was conducted, made obtaining consent for all patients impractical.

Open Access Statement: This is an Open Access article distributed in accordance with the Creative Commons Attribution-NonCommercial-NoDerivs 4.0 International License (CC BY-NC-ND 4.0), which permits the noncommercial replication and distribution of the article with the strict proviso that no changes or edits are made and the original work is properly cited (including links to both the formal publication through the relevant DOI and the license). See: https://creativecommons.org/licenses/by-nc-nd/4.0/.

\section{References}

1. Punglia RS, Bifolck K, Golshan M, et al. Epidemiology, Biology, Treatment, and Prevention of Ductal Carcinoma In Situ (DCIS). JNCI Cancer Spectr 2018;2:pky063.

2. Early Breast Cancer Trialists' Collaborative Group (EBCTCG); Correa C, McGale P, et al. Overview of the randomized trials of radiotherapy in ductal carcinoma in situ of the breast. J Natl Cancer Inst Monogr 2010;2010:162-77.

3. Barrio AV, Van Zee KJ. Controversies in the Treatment of Ductal Carcinoma in Situ. Annu Rev Med 2017;68:197-211.

4. Julien JP, Bijker N, Fentiman IS, et al. Radiotherapy in breast-conserving treatment for ductal carcinoma in situ: first results of the EORTC randomised phase III trial 10853. EORTC Breast Cancer Cooperative Group and EORTC Radiotherapy Group. Lancet 2000;355:528-33.

5. Fisher B, Dignam J, Wolmark N, et al. Lumpectomy and radiation therapy for the treatment of intraductal breast cancer: findings from National Surgical Adjuvant Breast and Bowel Project B-17. J Clin Oncol 1998;16:441-52.

6. Donker $\mathrm{M}$, Litière $\mathrm{S}$, Werutsky $\mathrm{G}$, et al. Breast-conserving treatment with or without radiotherapy in ductal carcinoma In Situ: 15-year recurrence rates and outcome after a recurrence, from the EORTC 10853 randomized phase III trial. J Clin Oncol 2013;31:4054-9.

7. Holmberg L, Garmo H, Granstrand B, et al. Absolute risk reductions for local recurrence after postoperative radiotherapy after sector resection for ductal carcinoma in situ of the breast. J Clin Oncol 2008;26:1247-52.

8. Cuzick J, Sestak I, Pinder SE, et al. Effect of tamoxifen and radiotherapy in women with locally excised ductal carcinoma in situ: long-term results from the UK/ANZ DCIS trial. Lancet Oncol 2011;12:21-9.

9. McGuire KP, Santillan AA, Kaur P, et al. Are mastectomies on the rise? A 13-year trend analysis of the selection of mastectomy versus breast conservation therapy in 5865 patients. Ann Surg Oncol 2009;16:2682-90.

10. Silverstein MJ, Barth A, Poller DN, et al. Ten-year results comparing mastectomy to excision and radiation therapy for ductal carcinoma in situ of the breast. Eur J Cancer 1995;31A:1425-7.

11. Ward EM, DeSantis CE, Lin CC, et al. Cancer statistics: Breast cancer in situ. CA Cancer J Clin 2015;65:481-95.

12. Narod SA, Sopik V. Is invasion a necessary step for 
metastases in breast cancer? Breast Cancer Res Treat 2018;169:9-23.

13. Silverstein MJ, Lagios MD, Craig PH, et al. A prognostic index for ductal carcinoma in situ of the breast. Cancer 1996;77:2267-74.

14. Cheng L, Al-Kaisi NK, Gordon NH, et al. Relationship between the size and margin status of ductal carcinoma in situ of the breast and residual disease. J Natl Cancer Inst 1997;89:1356-60.

15. Neri A, Marrelli D, Megha T, et al. "Clinical significance of multifocal and multicentric breast cancers and choice of surgical treatment: a retrospective study on a series of 1158 cases". BMC Surg 2015;15:1.

16. Bonnier P, Body G, Bessenay F, et al. Prognostic factors in ductal carcinoma in situ of the breast: results of a retrospective study of 575 cases. The Association for Research in Oncologic Gynecology. Eur J Obstet Gynecol Reprod Biol 1999;84:27-35.

17. Tot T. Clinical relevance of the distribution of the lesions in 500 consecutive breast cancer cases documented in large-format histologic sections. Cancer 2007;110:2551-60.

18. Tot T, Gere M, Pekár G, et al. Breast cancer multifocality, disease extent, and survival. Hum Pathol 2011;42:1761-9.

19. General Rules I SEER Training [Internet]. [cited 2021 May 1]. Available online: https://training.seer.cancer.gov/ collaborative/system/tnm/t/size/rules.html

20. O'Shaughnessy J. Extending survival with chemotherapy in metastatic breast cancer. Oncologist 2005;10 Suppl

Cite this article as: O'Keefe TJ, Harismendy O, Wallace AM. Histopathological growth distribution of ductal carcinoma in situ: tumor size is not "one size fits all”. Gland Surg 2022;11(2):307-318. doi: 10.21037/gs-21-599
3:20-9.

21. Narod SA, Iqbal J, Giannakeas V, et al. Breast Cancer Mortality After a Diagnosis of Ductal Carcinoma In Situ. JAMA Oncol 2015;1:888-96.

22. Tot T. DCIS, cytokeratins, and the theory of the sick lobe. Virchows Arch 2005;447:1-8.

23. Tot T, Pekár G, Hofmeyer S, et al. Molecular phenotypes of unifocal, multifocal, and diffuse invasive breast carcinomas. Patholog Res Int 2010;2011:480960.

24. Moore KH, Thaler HT, Tan LK, et al. Immunohistochemically detected tumor cells in the sentinel lymph nodes of patients with breast carcinoma: biologic metastasis or procedural artifact? Cancer 2004;100:929-34.

25. Bleiweiss IJ, Nagi CS, Jaffer S. Axillary sentinel lymph nodes can be falsely positive due to iatrogenic displacement and transport of benign epithelial cells in patients with breast carcinoma. J Clin Oncol 2006;24:2013-8.

26. Giannakeas V, Sopik V, Narod SA. Association of Radiotherapy With Survival in Women Treated for Ductal Carcinoma In Situ With Lumpectomy or Mastectomy. JAMA Network Open 2018;1:e181100.

27. O'Keefe TJ, Wallace AM. Surveillance, Epidemiology, and End Results program underestimates breast cancer-specific mortality after ductal carcinoma in situ diagnosis. Breast Cancer Res Treat 2020;182:761-2.

28. O'Keefe TJ, Harismendy O, Wallace AM. Large and diffuse ductal carcinoma in situ: potentially lethal subtypes of "preinvasive" disease. Int J Clin Oncol 2022;27:121-30. 